\title{
EXTENDED DEPTH-OF-FIELD USING ADJACENT PLANE DEBLURRING AND MPP WAVELET FUSION FOR MICROSCOPE IMAGES
}

\author{
Hyohoon Choi*, Samuel Cheng $\dagger$, Qiang Wu†, Kenneth R. Castleman $\dagger$, Alan C. Bovik $\ddagger$ \\ * Department of Biomedical Engineering, University of Texas, Austin, Texas \\ $\dagger$ Advanced Digital Imaging Research, LLC., 2450 South Shore Blvd., Suite 305, League City, Texas \\ $\ddagger$ Department of Electrical and Computer Engineering, University of Texas, Austin, Texas \\ Email: *hyohoon_choi@mail.utexas.edu, $\dagger\{$ samuelcheng,qwu,castleman $\} @$ adires.com \\ †bovik@ece.utexas.edu
}

\begin{abstract}
Imaging specimens thicker than the depth-of-field of a microscope produces poor quality images as only a portion of the specimen is in focus. Therefore, even in the best focused image, there are always objects that are out of focus and thus blurred. It is difficult to accurately measure the size, shape, and boundary of a blurred object. As a result, several optical sections are often required to estimate accurately the entire intensity distribution of the specimen. To overcome this problem, we introduce a novel method of extending the depth-offield by fusing several optical sections in the wavelet domain using multiscale point-wise product (MPP) criteria. Most existing fusion methods rely on criteria that are merely based on edges and do not distinguish signals from noise. However, our MPP criteria ensures that the signal content, rather than the noise, is collected. Instead of directly fusing optical sections, we preprocess the images by performing adjacent plane deblurring that removes blurred content and preserves the in-focus objects. The overall scheme provides superior quality images with extended depth-of-field and yet the fused images are insensitive to noise. The experimental results indicate both qualitatively and quantitatively that our approach outperforms existing schemes in the literature.
\end{abstract}

\section{INTRODUCTION}

Imaging thick specimens using a high power microscope results in narrow depth-of-field (DOF) and inevitably poses a significant problem when automatic analysis of images is desired. Objects outside the depth-of-field are blurred, which hinders the accurate analysis of the specimen. For example, to count the correct number of fluorescence in-situ hybridization (FISH) labeled dots, multiple optical sections need to be considered simultaneously because in any given optical section some dots are significantly blurred, or even invisible. Ana-

This work was supported in part by the NIH under the SBIR Grant 2R44RR016817-02A1. lyzing a stack of images is quite a challenge even with human assistance.

It is beneficial to have a large DOF such that the whole specimen is visible without blurring. The DOF can be increased either physically by changing the optics, which is extremely costly, or algorithmically by image fusion. Image fusion is a technique that combines several input images into a single composite image that contains most of the useful information. In order to achieve extended DOF by fusion, in-focus regions are collected from optical sections and put together to form one image. There are a number of fusion methods available, and they differ mainly by the region selection criteria $[1,2]$. One simple method is to average the input images, followed by sharpening. Although this method does not have any selection criteria, it is frequently used because of its simplicity. A proper selection criteria should collect regions with high saliency. Excluding those in the lowest frequency band, wavelet coefficients have high magnitudes where edges (abrupt changes) occur and have low magnitudes where pixel values are relatively constant. Utilizing this idea, images are transformed into the wavelet domain, and maximum wavelet coefficients are collected at every pixel among input images $[1,2]$. A composite image is created in the wavelet domain, and finally, the fused image can be obtained using the inverse wavelet transform. Obviously this method produces much better results than the simplistic averaging plus sharpening approach. However, artifacts usually exist in the fused image because the region selection criterion has no mechanism of distinguishing signals from noise.

We introduce a novel image fusion method based on multiscale pointwise product (MPP) criteria [3, 4]. It provides information regarding where the signal and noise are located. In order to extended the DOF, not only is the fusion process important, but the image acquisition and deblurring of optical sections are as well. None of the existing extended DOF methods perform deblurring prior to fusion. The deblurring process removes blurred content while preserving in-focus content. Thus the fused image with deblurring is sharper than 
that obtained without deblurring.

In Section 2, adjacent plane deblurring and MPP wavelet image fusion are discussed in detail. In Section 3, deblurring and MPP fusion results are shown along with example images. And we conclude the paper in Section 4.

\section{METHODS}

When a three dimensional specimen is imaged using a microscope, only regions inside the DOF are imaged sharply. On the other hand, the regions outside the DOF are blurred. This blurring can be modeled as a convolution with a defocus point spread function. The point spread function is a system's impulse response. It is a function of defocus distance, the distance from the object to the focal plane. Ideally if we captured and fused a stack of $N$ images with taken a distance DOF apart, the overall DOF of the fused image will be extended by $N$ times. Depending on the distribution of the specimen along the $z$-axis, the difference among adjacent images could be too small to be distinguishable by the human eye. Therefore, capturing images at every optical DOF represents overkill in most cases, and often only a small number of images is necessary to generate a decent fused image.

Once $N$ optical sections are obtained, adjacent plane deblurring can partially remove the defocused contents by utilizing two adjacent planes according to following equation [5].

$$
f_{j}=\alpha I_{j}-\beta\left(I_{j-1}+I_{j+1}\right) * P S F
$$

where $f_{j}$ is the deblurred image at the focus level $j$, and $I_{j}$ is an optical section obtained at the level $j$, and $P S F$ is the point spread function, and $*$ represents the convolution operation. The defocus distance for the point spread function is the distance between optical sections. The choice of the values for $\alpha$ and $\beta$ is critical in order to obtain the optimal deblurring result. These values are usually chosen heuristically. Here, we introduce a new way of calculating proper values for $\alpha$ and $\beta$. The basic idea is that $\alpha$ and $\beta$ should be chosen to remove the blurred contents in $I_{j}$. These contents may be better focused in the other two images and may appear sharply in either $I_{j-1}$ or $I_{j+1}$. Thus if we call a pixel value from a blurred region in $I_{j}$ as $x_{j}$ and pixel values in the same location in $I_{j-1}$ and $I_{j+1}$ as $x_{j-1}$ and $x_{j+1}$, pluging $x_{j-1}, x_{j}$, and $x_{j+1}$ in Eq. (1) should give zero or close to the background intensity. The same can be done in multiple locations. The optimum values for $\alpha$ and $\beta$ are found by solving these linear equations. Using this deblurring method, when $N$ images are captured, $N-2$ middle images are deblurred. Deblurred images are then fused by the following fusion method.

For fusion, deblurred images are decomposed by a type of overcomplete wavelet transform based on cubic spline [6]. Let $f$ be a deblurred image. The $j^{t h}$-level decomposition is
Table 1. FIR filters for decomposition and reconstruction

\begin{tabular}{c|c|c|c|c|c|c|c}
\hline taps & $h$ & $\tilde{h}$ & $g^{(1)}$ & $g^{(2)}$ & $\tilde{g}^{(1)}$ & $\tilde{g}^{(2)}$ & $u$ \\
\hline-4 & & & & & $1 / 256$ & & $1 / 256$ \\
\hline-3 & & & & & $9 / 256$ & $-1 / 128$ & $8 / 256$ \\
\hline-2 & $1 / 16$ & $1 / 16$ & & & $37 / 256$ & $-10 / 128$ & $28 / 256$ \\
\hline-1 & $1 / 4$ & $1 / 4$ & -1 & $1 / 2$ & $93 / 256$ & $-47 / 128$ & $56 / 256$ \\
\hline 0 & $3 / 8$ & $3 / 8$ & 1 & -1 & $-93 / 256$ & $-140 / 128$ & $70 / 256$ \\
\hline 1 & $1 / 4$ & $1 / 4$ & & $1 / 2$ & $-37 / 256$ & $-47 / 128$ & $56 / 256$ \\
\hline 2 & $1 / 16$ & $1 / 16$ & & & $-9 / 256$ & $-10 / 128$ & $28 / 256$ \\
\hline 3 & & & & & $-1 / 256$ & $-1 / 128$ & $8 / 256$ \\
\hline 4 & & & & & & & $1 / 256$ \\
\hline
\end{tabular}

performed as follows:

$$
\left\{\begin{array}{l}
S_{2^{j}} f=S_{2^{j-1}} f *(h, h)_{\uparrow 2^{j-1}} \\
W 1_{2^{j}} f=S_{2^{j-1}} f *\left(g^{(2)}, d\right)_{\uparrow 2^{j-1}} \\
W 2_{2^{j}} f=S_{2^{j-1}} f *\left(d, g^{(2)}\right)_{\uparrow 2^{j-1}} \\
W 3_{2^{j}} f=S_{2^{j-1}} f *\left(g^{(1)}, g^{(1)}\right)_{\uparrow 2^{j-1}},
\end{array}\right.
$$

where $S_{0} f=f$ and $I *(h, g)_{\uparrow 2^{j-1}}$ represent the separable convolution of the rows and columns of the image with 1-D filters, $[h]_{\uparrow 2^{j-1}}$ and $[g]_{\uparrow 2^{j-1}}$, respectively, and $[h]_{\uparrow m}$ represents the up-sampled sequence of the filter $\{h(n)\}$ by an integer factor $m$. The symbol $d$ denotes the Dirac filter with value one at the origin and zero for anywhere else.

The image is recovered by the following reconstruction formula:

$$
\begin{aligned}
S_{2^{j-1}} f= & W 1_{2^{j}} f *\left(\tilde{g}^{(2)}, u\right)_{\uparrow 2^{j-1}}+W 2_{2^{j}} f *\left(u, \tilde{g}^{(2)}\right)_{\uparrow 2^{j-1}} \\
& +W 3_{2^{j}} f *\left(\tilde{g}^{(1)}, \tilde{g}^{(1)}\right)_{\uparrow 2^{j-1}}+S 2_{2^{j}} f *(\tilde{h}, \tilde{h})_{\uparrow 2^{j-1}}
\end{aligned}
$$

where $u(j)=\left(1 /\left(2^{2 n+2}\right)\right)\left(\begin{array}{c}2 n+2 \\ j\end{array}\right), 0 \leq j \leq 2 n+2$, are the finite-impulse responses (FIRs) of the transfer function $U(\omega)=H^{2}(\omega)$. The values of $h$ and $g$ are shown in Table . 1. Further details are found in [4].

Our goal of image fusion is to merge the in-focus components from each deblurred images and reconstruct an image that contains most of the useful information. The in-focus regions are selected using MPP values. In wavelet domain, true signals has strong correlation across multiple decomposition scales whereas noise tends to be uncorrelated across scales. MPP is defined as

$$
p_{L}^{k} f(x, y)=\prod_{j=1}^{L} W_{2^{j}}^{k} f(x, y),
$$

where $k=1,2$, and 3 corresponds to the MPPs for the variation along different directions (horizontal, vertical, and diagonal) and $L$ is the number of decomposition levels. Since $p_{L}^{k} f(x, y)$ only reinforces the response from a true signal, its magnitude is high for a true signal (correlated across scales) but small for noise (uncorrelated across scales). Thus its magnitude naturally differentiates a signal from noise. A map is 
generated based on the comparison of MPP values across the input images at every pixel. Each pixel in the fusion map, $M(x, y)$, contains the index of the image that has the highest MPP magnitude. Since an object may occupy more than a single pixel, isolated pixels likely correspond to a selection error. Thus, it is useful to apply majority filtering on $M$ to eliminate isolated pixels. Wavelet coefficients are collected from corresponding images based on the map. Then the inverse wavelet transform of the composite wavelet coefficients brings the fused image, which is sharper and contains more useful information than any single optical section. The schematic diagram of our fusion process is shown in Fig. 1.

Sharpness increase is measured quantitatively. Since the lowest frequencies contribute to the global structure and noise tends to dominate the highest frequencies, it is the best to measure the mid-frequency information to quantify the sharpness. The sharpness is measured as follows.

$$
S=\frac{1}{N} \sum\left\{(I * h)^{2}+\left(I * h^{T}\right)^{2}\right\}
$$

where $h=[-1,-1,1,1]^{T}$, and $I$ is the image, and $N$ is the number of pixels, and $T$ represents the transpose operation of a matrix. The first term in Eq. (5), $(I * h)^{2}$, corresponds to the mid-frequencies of horizontal edges and the other term corresponds to the mid-frequencies of vertical edges. For color images, the sharpness for each color channel is calculated and the average is used to estimate the overall sharpness.

\section{RESULTS}

We have applied our method to images of FISH labeled chromosomes in interphase cell nuclei. When FISH labeled chromosomes are imaged under a fluorescence microscope, they appear as dots. One of the major difficulties of counting the dots is that not all dots appear in one optical section. Thus, by extending the DOF, we can visualize all the dots simultaneously in one image. Having all the dots in one single image is very useful for medical analysis, which we will not discuss in this paper.

Five images are captured and the middle three images are deblurred using adjacent plane deblurring. Then the deblurred images are fused using MPP criteria. For RGB color images, the same process is applied independently to each channel. However, since wavelet fusion tends to distort the color information when each color channel is processed independently, RGB images are first transformed into other color image formats such as YUV and HSI before fusion. Preliminary experimental results indicate similar performance between YUV and HSI color formats. As a future work, we will further investigate the effects of other color formats on various types of images.

Fig. 2 shows an example of the fusion process: (a), (b), and (c) are the original images; (d) shows the fusion result of original images; (e), (f), and (g) display the original images after deblurring; (h) shows the fusion result of the deblurred images. Interphase cell nuclei are stained with a blue dye, and chromosomes are stained with green and red dyes. Since dots are scattered farther apart than the DOF of the microscope, none of the original images as shown in Fig. 2 capture all the dots simultaneously. Fusion results in both cases, Fig. 2 (d) and (h), display all the dots clearly as if the DOF has been extended optically. Deblurred images show a significant improvement of contrast and quality compared to the original images. In our example, background intensity in green channel is significantly higher than other channels, and thus it makes signals from other channels not visible. Background elevation occurs when a long exposure time is used to capture weak signal, when unbound fluorophores are not washed thoroughly, and when the dark current in CCD is high. Since our deblurring method removes blurred contents along with low frequency components, slowly varying background in green channel is removed. Thus the signals from other channels are revealed. A caution needs to be taken when bright field images are deblurred. Since its signal is dark and background is bright, images should be inverted before the deblurring.

The sharpness value for Fig. 2 (d) and (h) are 511 and 1186, respectively. With deblurring, the sharpness value increases more than twice.

\section{CONCLUSION}

We introduced a new wavelet fusion method based on MPP criteria in order to obtain images with extended DOF. In conjunction with the MPP wavelet fusion, we have applied adjacent plane deblurring to remove out of focus content prior to the fusion. The main advantage of MPP based fusion over existing approaches is that it collects in-focus signals much more effectively since the MPP criteria can separate signal from noise. Thus our method is naturally more resilient to random noises than other approaches. We also proposed a new way in computing two essential parameters required for adjacent plane deblurring. Deblurring before the fusion increases contrast and sharpness significantly. The overall scheme provides superior quality microscope images with extended DOF while remaining insensitive to noise.

\section{REFERENCES}

[1] Hui Li, B.S. Manjunath, and S.K. Mitra, "Multi-sensor image fusion using the wavelet transform," Proceedings of 1st International Conference on Image Processing, vol. vol.1, pp. 51-55, 1994.

[2] B. Forster, D. Van De Ville, J. Berent, D. Sage, and M. Unser, "Extended depth-of-focus for multi-channel microscopy images: a complex wavelet approach," 2004 2nd IEEE International Symposium on Biomedical Imaging: Macro to Nano, vol. Vol. 1, pp. 660-, 2004. 


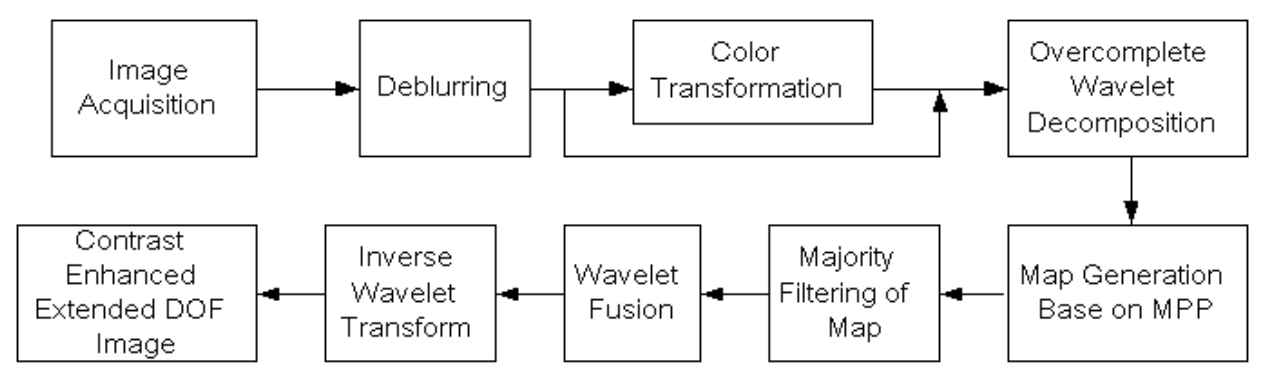

Fig. 1. Deblurring and MPP Wavelet Fusion Procedure

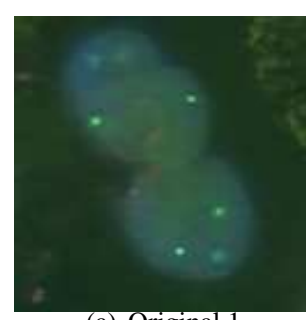

(a) Original 1

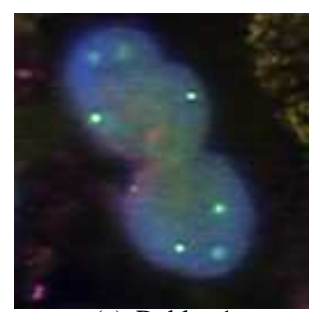

(e) Deblur 1

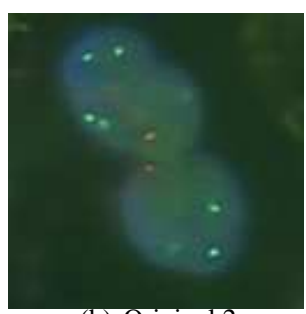

(b) Original 2

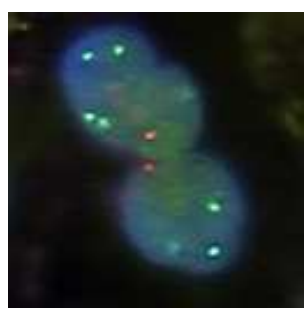

(f) Deblur 2

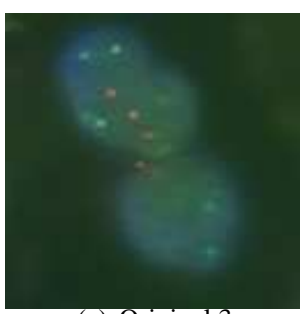

(c) Original 3

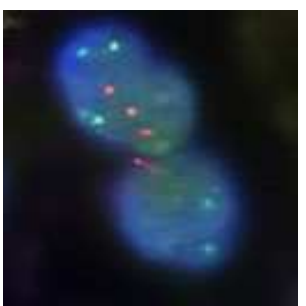

(g) Deblur 3

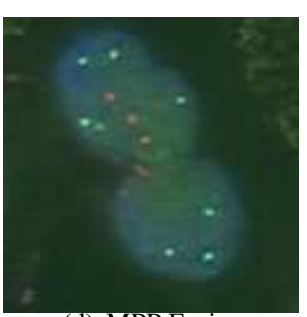

(d) MPP Fusion

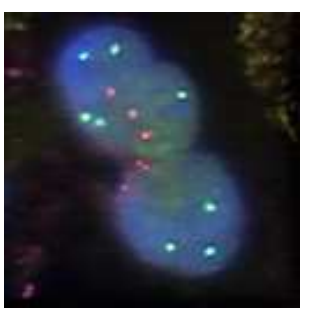

(h) MPP Fusion

Fig. 2. Deblurring and Fusion Result

[3] S. Mallat and S. Zhong, "Characterization of signals from multiscale edges," Pattern Analysis and Machine Intelligence, IEEE Transactions on, vol. 14, pp. 710-732, 1992.

[4] Yu-Ping Wang, Qiang Wu, K. R. Castleman, and Zixiang Xiong, "Chromosome image enhancement using multiscale differential operators," IEEE Transactions on Medical Imaging, vol. 22, no. 5, pp. 685-693, May 2003.

[5] Kenneth R. Castleman, Digital Image Processing, Prentice-Hall, Upper Saddle River, NJ, 1996.

[6] Yu-Ping Wang and S.L. Lee, "Scale-space derived from b-splines," IEEE Transactions on Pattern Analysis and Machine Intelligence, vol. 20, no. 10, pp. 1040-1055, Oct. 1998. 